\begin{tabular}{llll} 
Abstract P34 Table 1 & & & \\
\hline & Low risk & High risk & All patients \\
\hline Total & 86 & 212 & 298 \\
Pathological diagnosis achieved & 2 & 96 & 98 \\
Sarcoidosis & 0 & 46 & 46 \\
NSCLC & 1 & 16 & 17 \\
TB & 1 & 14 & 15 \\
SCLC & 0 & 8 & 8 \\
Non 1 ${ }^{\circ}$ lung cancer & 0 & 5 & 5 \\
Lymphoma & 0 & 7 & 7 \\
Other granulomatous disorder & 0 & 2 & 2 \\
False negative & 0 & 57 & 57 \\
Sarcoidosis & 0 & 46 & 46 \\
NSCLC & 0 & 2 & 2 \\
TB/NTM & 0 & 3 & 3 \\
Non 1 ${ }^{\circ}$ lung cancer & 0 & 1 & 1 \\
Lymphoma & 0 & 4 & 4 \\
Reactive lymphadenopathy & 84 & 59 & 143 \\
Negative predictive value & $100 \%$ & $49.1 \%$ & $71.5 \%$ \\
\hline
\end{tabular}

\section{P35 A SINGLE CENTRE PROSPECTIVE ANALYSIS OF THE EFFECT OF NEEDLE GAUGE ON EBUS-TBNA SENSITIVITY AND SAFETY}

FR Millar, J Gates, A Kumar, MK Menon, RB Banka. Department of Respiratory Medicine, King George Hospital, Barking, Havering and Redbridge NHS Trust, London, UK

\subsection{6/thoraxjnl-2017-210983.177}

Introduction EBUS-TBNA is the current gold standard technique for lymph node sampling in mediastinal lymphadenopathy. Traditionally our trust has used size 21 gauge needles for all EBUS-TBNA cases, however recently we have used 19 gauge needles in an effort to increase diagnostic yield. We present preliminary Results from a small, randomised, single centre study assessing the sensitivity and safety of 19 gauge EBUS needles versus 21 gauge EBUS needles in EBUS-TBNA.

Methods Patients attending for EBUS-TBNA were prospectively randomised to either 19 gauge or 21 gauge groups. Samples were sent for analysis and the pathologist was blinded to the needle size used. The primary outcomes measured were positive sampling of lymphoid tissue and diagnostic sampling. Complications including severe bleeding and pneumothorax were included in the analysis.
Results A total of 61 patients were enrolled in the study, with 32 assigned to the 21 gauge group and 29 assigned to the 19 gauge group. The average age was $57.5+/-6.8$ years and 53.2 $+/-5.4$ for the 21 gauge and 19 gauge groups respectively $(\mathrm{p} \geq 0.05)$. In the 21 gauge needle group lymphoid tissue was obtained in 27 of the 32 cases (84.38\%), whereas the 19 gauge needle group lymphoid tissue was obtained in 25 of 29 cases $(86.2 \%)(\mathrm{p} \geq 0.05)$. Diagnostic sampling was obtained in 20 of the $32(62.5 \%)$ cases in the 21 gauge needle group versus 19 of the 29 cases in the 19 gauge group (65.5\%) $(p \geq 0.05)$. None of our patients in either cohort suffered severe bleeding or pneumothorax.

Conclusions/Limitations Currently our study has shown no significant difference in either sensitivity or safety between 19 and 21 gauge EBUS-TBNA. However we recognise that as yet our study is under powered and continued enrolment of patients is required to obtain valid Conclusions Given that size 21 gauge needles confer a significant cost saving ( $£ 7800$ per annum) our trust are likely to continue using these needles if our preliminary Results are confirmed.

\section{P36 EBUS-TBNA DIAGNOSTIC YIELD CAN BE MAINTAINED WHEN PERFORMED BY A TRAINER SUPERVISING A SECOND OPERATOR TRAINEE}

${ }^{1} \mathrm{~A}$ Gupta, ${ }^{2} \mathrm{D}$ Nicoara, ${ }^{2} \mathrm{~A}$ Sajeed, ${ }^{2}$ RK Panchal, ${ }^{2} \mathrm{M}$ Tufail, ${ }^{2} \mathrm{~J}$ Bennett. ${ }^{1}$ University Hospitals of Leicester, Leicester, UK; ${ }^{2}$ Glenfield Hospital, Leicester, UK

\subsection{6/thoraxjnl-2017-210983.178}

Introduction Endobronchial ultrasound-guided transbronchial needle aspiration (EBUS-TBNA) facilitates diagnosis of hilar and mediastinal lymphadenopathy. The procedure usually requires two operators; one guiding the bronchoscope and other undertaking needling for biopsy. British Thoracic Society Quality Standards recommend a diagnostic yield of $88 \%$. Increased training and experience has been shown to improve diagnostic yield over time. ${ }^{1}$ Services face a potential conflict between delivering a high quality service, as evidenced by high diagnostic yield, and training specialty registrars. Our EBUS-TBNA trainers have trained 35 trainees since 2011. We evaluated EBUS diagnostic yield at a tertiary centre with procedures carried out by trainer and trainee operators.

Methods Retrospective analysis of two operator EBUS-TBNA procedures from March 2011 to December 2016 was carried out. Data was collected on gender, age, procedure date and clinicians

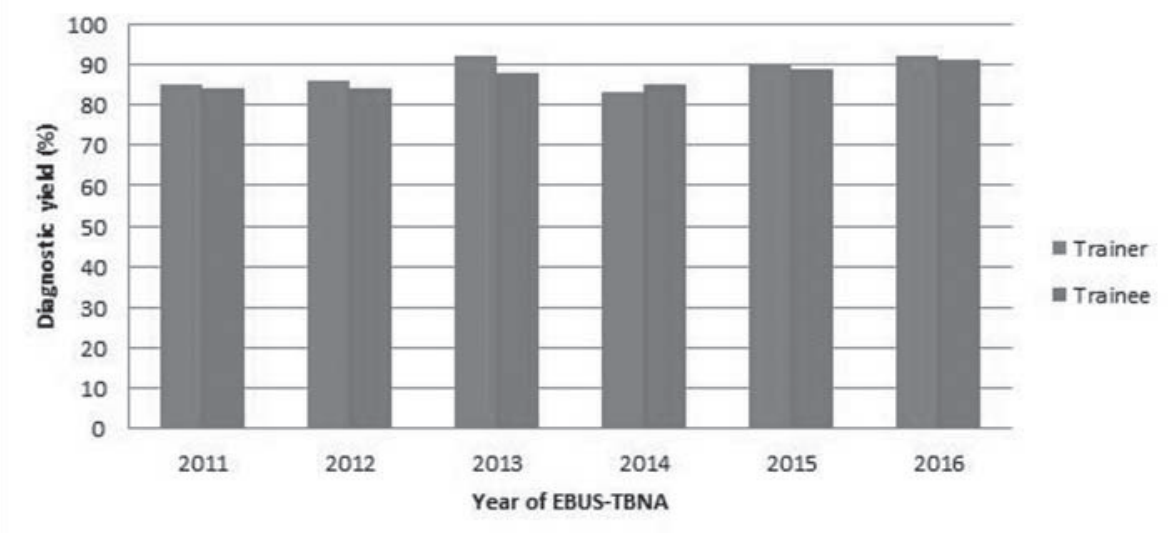

Abstract P36 Figure 1 Diagnostic yield of EBUS-TBNA for trainers vs trainees from 2011-2016. 
involved in the procedure. Overall diagnostic yields were calculated for trainers compared to trainees for both manoeuvring the bronchoscope and needling. Diagnostic rates according to year of EBUS-TBNA for trainers vs trainees were also calculated. MannWhitney $U$ test was used to check for differences in hit-rates for trainers vs trainees between the two components of the procedure, whilst Kruskal Wallis test was used to assess difference in diagnostic yield between 2011-2016.

Results 1083 patients underwent EBUS-TBNA with mean age 61 years (SD +/-14), 464 (43\%) were female. The overall diagnostic rate was $88 \%$, with 479 (44\%) malignant, 212 (20\%) granulomatous, 262 (24\%) benign and 130 (12\%) nondiagnostic. Trainees manoeuvred the bronchoscope for 577 $(53 \%)$ and needled for 461 (43\%) patients. There were no differences in diagnostic yields between trainers compared to trainees for manoeuvring [ $88 \%$ vs $88 \% \quad(p=0.81)]$ nor for needling [ $88 \%$ vs $88 \%(\mathrm{p}=0.84)$. There were also no significant differences in diagnostic yield between years (figure1).

Conclusion In our institution we can teach multiple trainees whilst maintaining high diagnostic yield in two operator EBUS-TBNA for both manoeuvring the bronchoscope and needling when one operator is fully trained.

\section{REFERENCE}

1. Fuso, et al. Influence of the learning effect on the diagnostic yield of endobronchial ultrasound-guided transbronchial needle aspiration of mediastinal and hilar lymph nodes. Journal of Bronchology \& Interventional Pulmonology 2017;24 (3):193-199.

\section{P37 THE CLINICAL UTILITY OF RAPID ON-SITE EVALUATION (ROSE) IN THE DIAGNOSIS OF NON-MALIGNANT GRANULOMATOUS MEDIASTINAL LYMPHADENOPATHY FOLLOWING ENDOBRONCHIAL ULTRASOUND (EBUS)}

J Capps, K Heyes, S Bailey, T Gorsuch, M Woodhead, D Shelton, D Rana, N Narine, H AlNajjar. Central Manchester Foundation Trust, Manchester, UK

\subsection{6/thoraxjnl-2017-210983.179}

Introduction EBUS guided trans-bronchial needle aspiration with ROSE ensures adequacy of specimen samples and provides preliminary cytological diagnosis. Few studies have explored the utility of ROSE in granulomatous mediastinal lymphadenopathy. This retrospective study looks to further assess the validity of ROSE in the setting of non-malignant granulomatous disease.

Methods We reviewed a prospectively maintained database of ROSE and laboratory cytology Results for all EBUS procedures performed during a 12 month period from 1 st January to 31 st December 2015 at our institute. We included all patients who had granuloma (including probable or possible granuloma) identified at ROSE or final cytology analysis, or both. We then reviewed clinico-radiological data to ascertain the final diagnosis and excluded those patients with malignant disease.

Results During the study period, 366 EBUS were performed, with granuloma identified in 51 patients. Three patients were found to have malignancy and were excluded therefore 48 were included in the final analysis. The final diagnoses for the 48 patients are shown in Table 1. Patients with TB were more likely to have at least one granuloma at ROSE (84\%) than patients with sarcoidosis (67\%). Patients with granuloma identified at ROSE had a slightly lower number of nodes sampled per patient compared to those with no granuloma at ROSE (mean 1.8 vs 2.4 nodes per patient). The positive predictive value of ROSE for granuloma in our cohort was $100 \%$, with a sensitivity of $71 \%$. This is comparable to other studies.

Conclusions In our cohort of patients, ROSE had a high positive predictive value and a sensitivity of over $70 \%$ for the diagnosis of granuloma in non-malignant disease. Our Results suggest that with the use of ROSE fewer nodes are sampled which may reduce procedure time and potential complications. This study is limited due to the small sample size but supports the use of ROSE in this context. We plan to carry out further work with larger data sets, and to look at the characteristics of those subsequently diagnosed with sarcoidosis or tuberculosis.

\begin{tabular}{lllll}
\multicolumn{2}{l}{ Abstract P37 Table 1} \\
\hline & \multicolumn{2}{l}{ Granuloma at ROSE } & \multicolumn{2}{l}{ Granuloma at final cytology } \\
\hline Final diagnosis & N & $\%$ & N & $\%$ \\
\hline Sarcoidosis & 18 & 38 & 27 & 56 \\
\hline TB (all) & 16 & 33 & 19 & 40 \\
$\quad$ TB (culture positive) & 5 & 10 & 8 & 17 \\
$\quad$ TB (culture negative) & 11 & 23 & 11 & 23 \\
Reactive & 0 & 0 & 2 & 4 \\
Total & 34 & 71 & 48 & 100 \\
\hline
\end{tabular}

\section{P38 EBUS-TBNA IN LUNG CANCER - CAN WE SIMPLIFY DIAGNOSIS AND STAGING IN A SINGLE PROCEDURE?}

${ }^{1}$ LM Santos, ${ }^{2} \mathrm{D}$ Jaramillo, ${ }^{2} \mathrm{M}$ Jacomelli, ${ }^{2}$ VR Figueiredo. ${ }^{1}$ Pneumology Unit, Hospitais da Universidade de Coimbra, Centro Hospitalar e Universitário de Coimbra, Coimbra, Portugal; ${ }^{2}$ Respiratory Endoscopy Service, Pulmonary Division of Heart Institute (InCor), Hospital das Clínicas, Faculdade de Medicina da Universidade de São Paulo, Coimbra, Portugal

10.1136/thoraxjnl-2017-210983.180

Background Lung cancer(LC) is the leading cause of worldwide cancer-related deaths and its accurate diagnosis and staging is crucial to guide appropriate treatment and prognosis. ${ }^{1}$ EBUS-TBNA is a minimally invasive standard procedure for staging that has proven to be useful in diagnosis too, allowing a complete characterisation of the disease in a single procedure and, thus, decreasing time-to-treatment. ${ }^{2}$

Aim To evaluate the role of EBUS-TBNA as initial technique for simultaneous diagnosis and staging in patients with suspected LC and calculate its sensitivity, specificity, positive and negative predictive values.

Methods Retrospective study of all patients with suspected LC in computed tomography(CT) or positron-emission tomography(PET)/CT submitted to EBUS-TBNA for simultaneous diagnosis and staging, from September/2011 to February/2017. Results of EBUS-TBNA were compared to surgical ones, when patients were subsequently submitted to surgery, or to clinical and radiological follow-up.

Results Patients included( $\mathrm{n}=62)$ had mean age of 68 years $(\mathrm{SD}=10)$ and $66.1 \%$ were male. Smoking history was present in $53.2 \%$ and history of extrathoracic malignant disease in $17.7 \%$. Mean diameter of pulmonary lesions in CT or PET/CT was $37.2 \mathrm{~mm}(\mathrm{SD}=21 \mathrm{~mm})$ and in $64.5 \%$ of cases were associated to lymphadenopathy. $59.7 \%(\mathrm{n}=48)$ of patients were diagnosed with LC, $77.1 \%(n=37)$ of them by EBUS-TBNA, $8.3(n=4)$ by other bronchoscopic methods at same time of EBUS and 14.6\% $(n=7)$ needed surgical biopsy. LC diagnosed by EBUS-TBNA were $59.5 \%$ adenocarcinoma, $16.2 \%$ squamous, $21.6 \%$ small cell and $2.7 \%$ 